\title{
Robot-assisted Ivor-Lewis esophagectomy with intrathoracic robot-sewn anastomosis
}

\author{
Runsen Jin*, Jie Xiang*, Dingpei Han, Yajie Zhang, Hecheng Li \\ Department of Thoracic Surgery, Ruijin Hospital, Shanghai Jiao Tong University School of Medicine, Shanghai 200025, China \\ *These authors contributed equally to this work. \\ Correspondence to: Professor Hecheng Li. Department of Thoracic Surgery, Ruijin Hospital, Shanghai Jiao Tong University School of Medicine, \\ 197 Ruijin 2nd Road, Shanghai 200025, China. Email: lihecheng2000@hotmail.com.
}

\begin{abstract}
This video clip demonstrated a performance of robot-assisted Ivor-Lewis esophagectomy with intrathoracic robot-sewn anastomosis. The patient had an esophageal mass located approximately $33 \mathrm{~cm}$ away from incisor, and robot-assisted Ivor-Lewis esophagectomy was applied for him. Importantly, a double-layer esophago-gastric anastomosis was made by robotic hand-sewn suture. Our early experience demonstrated that the robot-sewn intrathoracic anastomosis is feasible and safe with a lower complication rate and the absence of anastomotic leakage.
\end{abstract}

Keywords: Robot-assisted; Ivor-Lewis esophagectomy (ILE); robot-sewn anastomosis

Submitted Apr 16, 2017. Accepted for publication Aug 03, 2017.

doi: $10.21037 /$ jtd.2017.09.96

View this article at: http://dx.doi.org/10.21037/jtd.2017.09.96

\section{Introduction}

Surgery is the priority treatment of esophageal cancer (1). Esophagectomy is technically challenging and is associated with high morbidity and mortality rates. Efforts to reduce these rates have spurred the adoption of minimally invasive techniques (2). The limitations of this approach, such as the two-dimensional (2D) view or movement restrictions, can, however, make a complex procedure such as esophagectomy difficult. Robotic systems have been designed to overcome some of these disadvantages, providing an amplified threedimensional (3D) view and a greater degree of freedom of movement, thanks to the articulated instruments (3). Most of the published reports on robotic esophagectomy describe two types of anastomosis including cervical or intrathoracic anastomosis by using the suturing technique $(4,5)$.

We have performed robot-assisted Ivor-Lewis esophagectomy (RA-ILE) since 2015 and have done approximate 50 cases. Here, for the first time in China, we report a case of the RA-ILE with intrathoracic robotsewn anastomosis, focusing on technical details with a video presented. Our initial experience suggest that the reported technique is safe and satisfies the oncological principles.

\section{Clinical data}

A 50-year-old man was admitted to our hospital with a 1-month history of abdominal distension and sour regurgitation. There was no obvious dysphagia retrosternal pain, or weight loss. Esophagogastroscopy identified a protrude type of mass located approximately $33 \mathrm{~cm}$ away from incisor, the pathological biopsy result was poorly differentiated carcinoma. Enhanced CT scan revealed a thick wall of the distal thoracic esophagus with no obvious metastases or enlarged lymph nodes (Figure 1). Physical examination was negative. The patient had a smoking and alcohol intake history of more than 20 years and the body mass index (BMI) is $24.57 \mathrm{~kg} / \mathrm{m}^{2}$. His cardiopulmonary function and laboratory examinations were normal. He had no past medical history.

\section{Operative techniques}

After the induction of general anesthesia, the patient was placed in a supine position. Then we placed three $8-\mathrm{mm}$ ports for the robotic arms and two 12-mm trocars for camera and the assistant (Figure 2). The robot is docked 


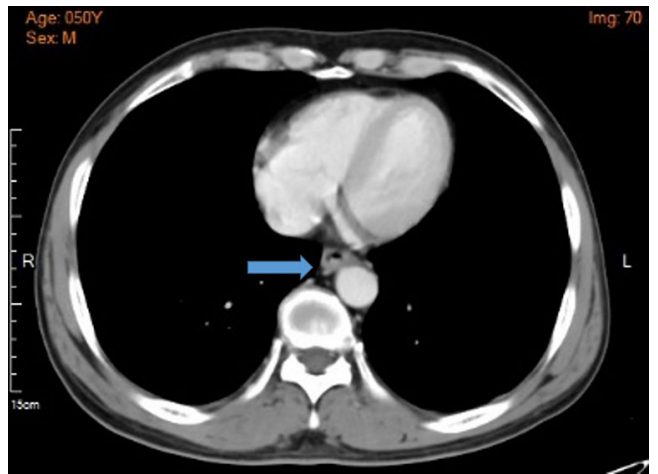

Figure $1 \mathrm{CT}$ scan of the patient demonstrated a mass located in the distal third of the esophagus (arrow) and the mediastinal lymph node is negative. CT, computed tomography.

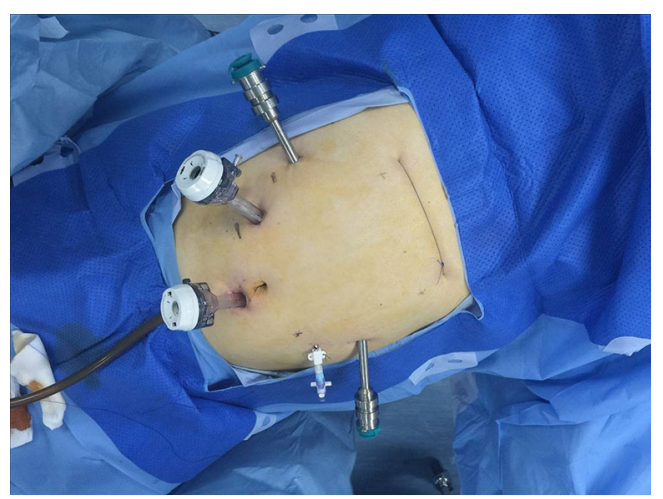

Figure 2 Ports for abdominal phase.
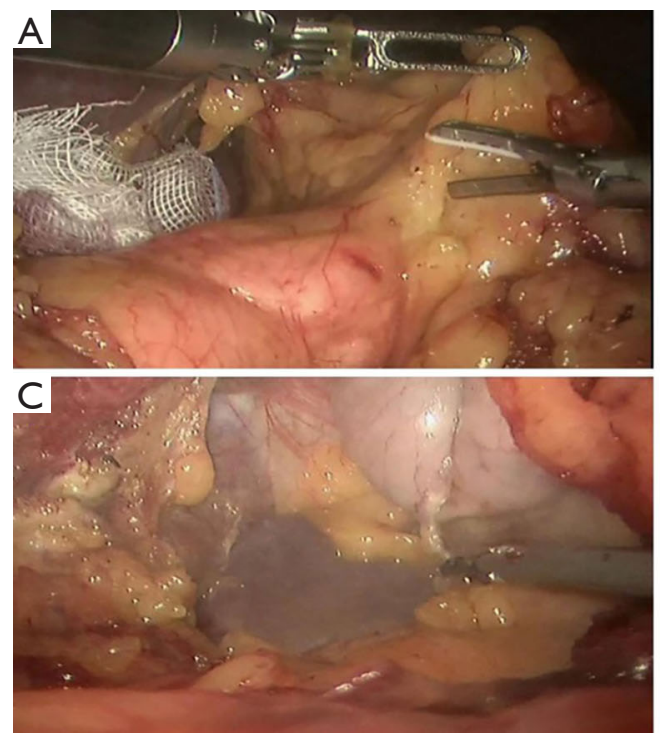

coming from the head of the patient. The $2 \#$ arm was connected with bipolar cautery grab and the $1 \#$ arm was connected with an ultrasound knife.

After hanging the liver with purse string, a radical "en bloc" lymphadenectomy was done along common hepatic artery, celiac trunk, and origin of splenic artery. Then left gastric vessels were dissected and interrupted. The adhesion between the stomach wall and pancreas was dissected, followed by cutting the gastro-colic ligament, splenogastric ligament, left gastro-epiploic and the short gastric vessels. In the end, a gastric tube was tailored using a stapling device (Figure 3).

Once the abdominal phase was completed, the patient is positioned in the left lateral decubitus position, tilted $45^{\circ}$ towards the prone position under double lumen endotracheal intubation. The first $12-\mathrm{mm}$ trocar was inserted at the right 5 th intercostal space (ICS) on the anterior axillary line which was used for the camera. The right robotic $8-\mathrm{mm}$ trocar was in the 8 th ICS and the left robotic $8-\mathrm{mm}$ trocar was in the 3 th ICS anterior to the scapular rim under direct vision. The two thoracic assisting ports in the 4th and 7th ICS were also indicated (Figure 4).

In this video, the procedures were as followed (Figure 5):

(I) The procedure was started from the upper mediastinum. Lymph nodes with their associated fat pads around the right recurrent laryngeal nerves were dissected completely;

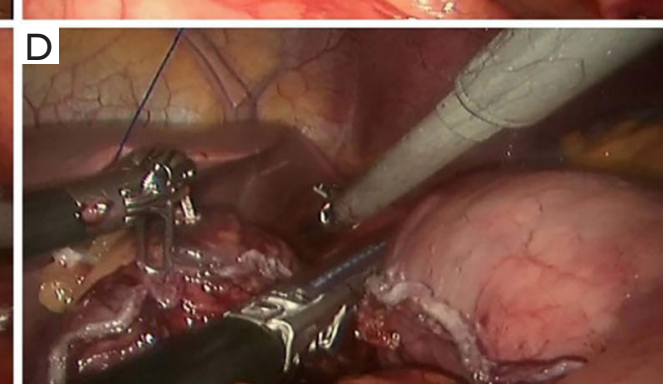

Figure 3 Main surgical procedures for abdominal phase. (A) Radical "en bloc" lymphadenectomy; (B) dissection of the left gastric vessels; (C) dissection of splenogastric ligament; (D) making gastric tube using a stapling device. 


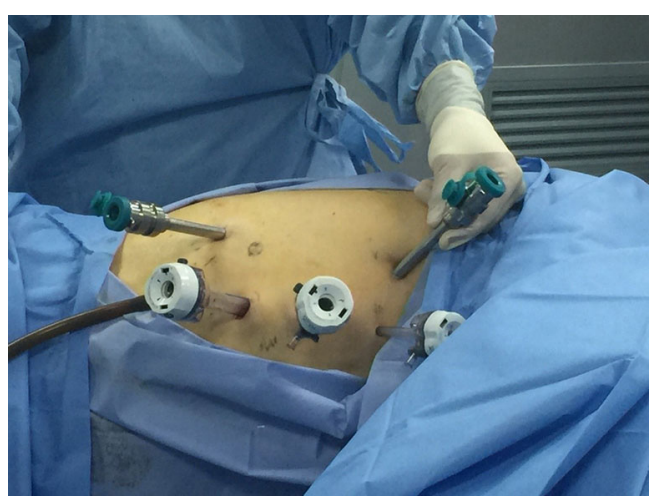

Figure 4 Ports for thoracic phase (5, 3, 8, 4, 7 ICS). ICS, intercostal space.

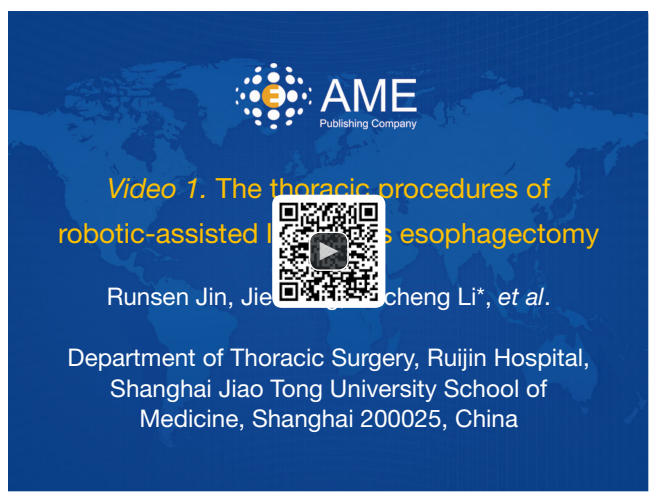

Figure 5 The thoracic procedures of robotic-assisted Ivor-Lewis esophagectomy (6). This video presents the thoracic procedures of robotic-assisted Ivor-Lewis esophagectomy, including complete mediastinal lymph nodes dissection, esophagus "en bloc" dissection, double-layer esophago-gastric anastomosis, etc.

Available online: http://www.asvide.com/articles/1839

(II) The azygos vein was dissected and divided with a stapling device;

(III) A complete lymph nodes dissection was carried out on sub-carinal region, right and left bronchus;

(IV) The esophagus was dissected "en bloc" with the mediastinal lymphatic tissue;

(V) The lymph nodes around the left recurrent laryngeal nerves were dissected completely with the help of a gauze suspending the esophagus;

(VI) The esophagus was cut off $5 \mathrm{~cm}$ above the lesion with a stapling device;

(VII) The gastric tube was carefully pulled up inside the chest avoiding rotation or traction, then the transection of gastric fundus was completed by stapler;
(VIII)A double-layer esophago-gastric anastomosis was made with a 3-0 absorbable barbed sutures by robotic hand-sewn suture: the posterior wall was continuously sutured for mucous layer and seromuscular layer; interrupted suture was applied for the anterior mucous layer, the seromuscular layer was then sutured continuously.

A gastric tube is placed inside the stomach. Finally, the anastomosis was checked by gastroscopy and a nasointestinal tube was also placed at the same time. Chest cavity drainage tube placed in the 8th ICS and negative suction drainage tube placed in the 10th ICS.

The postoperative treatments were anti-inflammation, enteral nutrition and phlegm-resolving, etc. The chest cavity drainage tube was withdrawn 2 days after surgery. The patient started a liquid diet on the 6th postoperative day. The patient was discharged on the 8 th postoperative day and could have some semi-liquid diet. No complication happened at the time of hospitalization. The postoperative pathology was poorly differentiated squamous cell carcinoma, tumor size $2.0 \mathrm{~cm} \times 2.0 \mathrm{~cm} \times 0.8 \mathrm{~cm}$, negative surgical margin, 29 lymph nodes were removed, with $1 / 2$ metastasis of periesophageal lymph nodes.

\section{Comments}

The first robotic-assisted minimally invasive esophagectomy (RAMIE) in the world was reported in 2003 by Dr. Horgan (7). In the past decades, the researches focused on RAMIE were fewer because of the operative difficulties of minimally invasive esophagectomy. There is still no standard surgical procedure for esophagectomy. It was reported that the cervical anastomosis is preferred by most surgeon over the intrathoracic $(8,9)$. However, it has been demonstrated that cervical anastomosis is associated with a higher rate of a higher percentage of leaks, stenosis and injuries to the recurrent laryngeal nerve, as well as postoperative dysphagia, and respiratory complications $(10,11)$. As a result, the Ivor-Lewis technique is increasingly being used especially for middle and lower esophageal cancer (12). RA-ILE was developed later than the McKeown approach, because of the greater complexity of performing a minimally invasive intra-thoracic anastomosis using stapler devices, as the insertion and correct positioning of staplers into the chest is very difficult and restricted by the rigidity of ICSs (13).

Robotic systems provide an amplified 3D view and a greater degree of freedom of movement, which make it easier for hand-sewn anastomosis. It was reported that 
RA-ILE with double-layer hand-sewn chest anastomosis has a lower complication rate $(6.3 \%)$ and the absence of anastomotic leakage in a series of 16 patients $(14,15)$. We have started performing RA-ILE with intrathoracic robot-sewn anastomosis recently. In this video, we presented technical details of hand-sewn anastomosis. Our experience demonstrated that the robot-sewn intra-thoracic anastomosis is feasible and safe. Further studies are needed to prove whether this procedure could provide long-term benefits to patients with esophageal cancer.

\section{Acknowledgements}

Funding: This study was supported by the grant from Shanghai Municipal Education Commission-Gaofeng Clinical Medicine Grant Support (No. 20172005) and Shanghai Municipal Commission of Health and Family Planning Outstanding Academic Leaders Training Program (No. 2017BR055).

\section{Footnote}

Conflicts of Interest: The authors have no conflicts of interest to declare.

Informed Consent: Written informed consent was obtained from the patient for publication of this manuscript and any accompanying images.

\section{References}

1. Dimick JB, Goodney PP, Orringer MB, et al. Specialty training and mortality after esophageal cancer resection. Ann Thorac Surg 2005;80:282-6.

2. Yerokun BA, Sun Z, Yang CJ, et al. Minimally Invasive Versus Open Esophagectomy for Esophageal Cancer: A Population-Based Analysis. Ann Thorac Surg 2016;102:416-23.

3. Ruurda JP, van der Sluis PC, van der Horst S, et al. Robotassisted minimally invasive esophagectomy for esophageal cancer: A systematic review. J Surg Oncol 2015;112:257-65.

4. Park SY, Kim DJ, Yu WS, et al. Robot-assisted thoracoscopic esophagectomy with extensive mediastinal lymphadenectomy: experience with 114 consecutive patients with intrathoracic esophageal cancer. Dis Esophagus 2016;29:326-32.
5. Hodari A, Park KU, Lace B, et al. Robot-Assisted Minimally Invasive Ivor Lewis Esophagectomy With Real-Time Perfusion Assessment. Ann Thorac Surg 2015;100:947-52.

6. Jin R, Xiang J, Li H, et al. The thoracic procedures of robotic-assisted Ivor-Lewis esophagectomy. Asvide 2017;4:520. Available online: http://www.asvide.com/ articles $/ 1839$

7. Horgan S, Berger RA, Elli EF, et al. Robotic-assisted minimally invasive transhiatal esophagectomy. Am Surg 2003;69:624-6.

8. Boone J, Livestro DP, Elias SG, et al. International survey on esophageal cancer: part I surgical techniques. Dis Esophagus 2009;22:195-202.

9. Enestvedt CK, Perry KA, Kim C, et al. Trends in the management of esophageal carcinoma based on provider volume: treatment practices of 618 esophageal surgeons. Dis Esophagus 2010;23:136-44.

10. Kassis ES, Kosinski AS, Ross P Jr, et al. Predictors of anastomotic leak after esophagectomy: an analysis of the society of thoracic surgeons general thoracic database. Ann Thorac Surg 2013;96:1919-26.

11. Atkins BZ, Shah AS, Hutcheson KA, et al. Reducing hospital morbidity and mortality following esophagectomy. Ann Thorac Surg 2004;78:1170-6; discussion 1170-6.

12. Bizekis C, Kent MS, Luketich JD, et al. Initial experience with minimally invasive Ivor Lewis esophagectomy. Ann Thorac Surg 2006;82:402-6; discussion 406-7.

13. Bongiolatti S, Annecchiarico M, Di Marino M, et al. Robot-sewn Ivor-Lewis anastomosis: preliminary experience and technical details. Int J Med Robot 2016;12:421-6.

14. Cerfolio RJ, Bryant AS, Hawn MT. Technical aspects and early results of robotic esophagectomy with chest anastomosis. J Thorac Cardiovasc Surg 2013;145:90-6.

15. Trugeda S, Fernández-Díaz MJ, Rodríguez-Sanjuán JC, et al. Initial results of robot-assisted Ivor-Lewis oesophagectomy with intrathoracic hand-sewn anastomosis in the prone position. Int J Med Robot 2014;10:397-403.

Cite this article as: Jin R, Xiang J, Han D, Zhang Y, Li H. Robot-assisted Ivor-Lewis esophagectomy with intrathoracic robot-sewn anastomosis. J Thorac Dis 2017;9(11):E990-E993. doi: 10.21037/jtd.2017.09.96 